\title{
Advancing Creative Visual Thinking with Constructive Function-based Modelling
}

\author{
Alexander Pasko and Valery Adzhiev \\ The National Centre for Computer Animation, \\ Bournemouth University, Bournemouth, UK
}

apasko@bournemouth.ac.uk, vadzhiev@bournemouth.ac.uk

\author{
Evgeniya Malikova and Victor Pilyugin \\ The National Research Nuclear University "MEPhl", \\ Moscow, Russia
}

\author{
malikova@sv-journal.com, pilyugin@sv-journal.com
}

\section{Executive Summary}

Modern education technologies are destined to reflect the realities of a modern digital age. The juxtaposition of real and synthetic (computer-generated) worlds as well as a greater emphasis on visual dimension are especially important characteristics that have to be taken into account in learning and teaching. We describe the ways in which an approach to constructive shape modelling can be used to advancing creative visual thinking in artistic and technical education. This approach assumes the use of a simple programming language or interactive software tools for creating a shape model, generating its images, and finally fabricating a real object of that model. It can be considered an educational technology suitable not only for children and students but also for researchers, artists, and designers. The corresponding modelling language and software tools are being developed within an international HyperFun Project. These tools are easy to use by students of different age, specialization and abilities, and can easily be extended and adapted for various educational purposes in different areas.

We applied the theoretical framework and software tools at different levels of education starting from elementary schools to doctoral thesis research in various areas related to artistic design and animation, computer graphics, programming languages, software development, and experimental and theoretical physics. In the process, the students learn how rather abstract mathematical expressions result in creating visually and meaningfully appealing computer-generated artefacts of a different nature that can eventually be fabricated as real objects using 3D printing means. Several application case studies in various areas of art, design, and technical education from different

Material published as part of this publication, either on-line or in print, is copyrighted by the Informing Science Institute. Permission to make digital or paper copy of part or all of these works for personal or classroom use is granted without fee provided that the copies are not made or distributed for profit or commercial advantage AND that copies 1) bear this notice in full and 2) give the full citation on the first page. It is permissible to abstract these works so long as credit is given. To copy in all other cases or to republish or to post on a server or to redistribute to lists requires specific permission and payment of a fee. Contact Publisher@,InformingScience.org to request redistribution permission. educational institutions and countries are presented. The obtained practical experience shows that this approach can be mastered and appreciated by students at different levels of education as an activity stimulating and supporting their creative thinking. The social context of the learning process allowing for collaborative work is also worth of noting. The presented case-studies demonstrate 
that seemingly complex modern means of geometric modelling and fabrication can easily be mastered not only by students oriented toward computer specialties but also by children (including those with disabilities) and artists.

Keywords: Interactive geometric modeling, Computer animation, Scientific visualization, 3D printing, Digital fabrication, Kinect, Function Representation FRep, HyperFun, Constructionism learning theory, Visual thinking.

\section{Introduction}

Visual thinking or human operating with mental images is strongly imbedded in modern culture through education, media, and entertainment. A new generation of educational technology is necessary with a particular emphasis on visual thinking to stimulate and facilitate creativity. Computer support of visual thinking remains on the ideology platform built more than 30 years ago. Computer graphics tools as the basis of this support were originally oriented to producing eye pleasing pictures. Nowadays real and synthetic worlds not only exist in parallel, but interact and interpenetrate each other. Examples of such interactions are areas such as 3D Face Recognition that performs automatic construction of an appropriate 3D model from a real face, Augmented Reality that suggests manipulating 3D objects through attached markers and combination of real time web camera view with 3D model in one imaginary scene, Digital Fabrication that brings 3D models to real world by 3D printing means, and modern cinematography techniques allowing to mix actors with synthetic characters generated by $3 \mathrm{D}$ computer animation means. This challenge has to be adequately addressed by new generation computer technologies, which require rethinking of the entire process ("pipeline") starting from real objects, their mental visualization and abstraction, mathematical description, computer representation, interactive manipulation with the model, generation of images, fabrication and utilization of new tangible objects thus creating augmented worlds. These worlds including real and virtual components can be experienced, explored, and modified.

Currently dominating geometric models in computer graphics are 2D vector graphics, 3D polygonal surfaces, and parametric surfaces. The problems with these models are well-known: nonprecise shape and visual properties definitions, growing memory consumption, limited complexity, topological ambiguities, and others. Such a geometric representation was able to be handled by computers 30-40 years ago. Modern multiprocessor computers are able to deal with much more complex data structures and mathematical models, which were previously unimaginable.

The ability to process and the necessity for compact precise models with unlimited complexity have resulted in the development of the new paradigm of procedural modelling and rendering, where the object's geometric shape and properties are evaluated upon request using procedural rules. One of the approaches to procedural modelling is to evaluate a set of real functions (or a vector-function) representing the shape and other object properties at the given point. A constructive approach to the creation of such function evaluation procedures for geometric shapes was proposed and called the Function Representation (FRep) in Pasko, Adzhiev, Sourin, and Savchenko (1995) and then extended in Pasko, Adzhiev, Schmitt, and Schlick (2001) to the case of point attribute functions representing such object properties as material, colour, transparency, and others. The main purpose of this approach is the creation of complex models from simple building blocks using operations similar to a model assembly in LEGO (see the illustration in Figure 1). While the user operates on the high-level of abstraction, a modelling system maintains the final function evaluation procedure for the modeled object. 


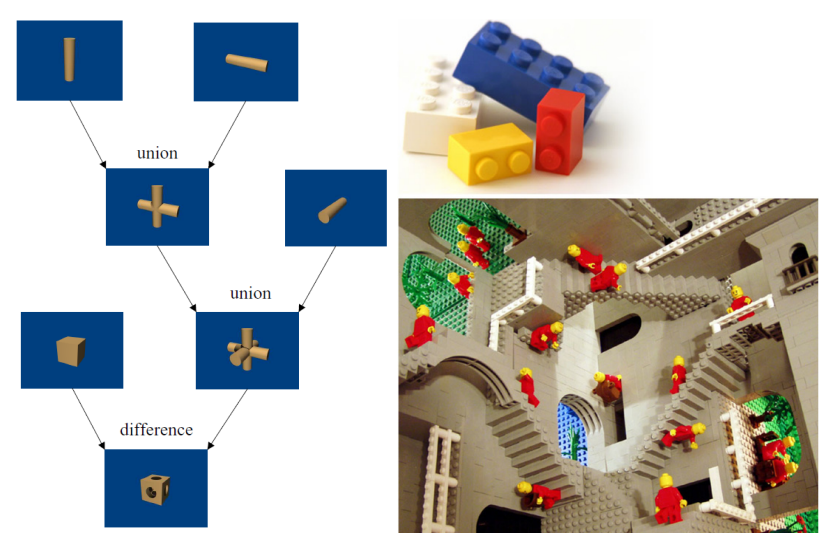

Figure 1: Constructive tree structure reflecting the logic of the object assembly (left), simple LEGO building blocks-primitives (top right), and a LEGO model assembled from those primitives (bottom right.) Source: nOmArch, 2009

It is worth noting that this approach corresponds to the constructionism learning theory introduced by Seymour Papert (Papert \& Harel, 1991). Application of this theory using computer technologies in educational practice is not always easy (Beynon \& Roe, 2004). It is well known that constructivist thinking is the basis of the LEGO construction toys which enable children to learn notions that were considered as too complex for them. We have been developing virtual modelling and graphics tools rather than physical ones providing an extendible set of "building blocks", which are deformable and modifiable on the fly. This approach assumes the model creation using a simple programming language or interactive tools with subsequent generation of its images, and finally fabricating a tangible replica of the created model. We believe that such an approach is of interest as an educational technology suitable not only for children and students, but also for researchers, artists, and designers. Earlier applications of this technology in education were reported in (Pasko \& Adzhiev, 2009). In this paper, we would like to present further experience and case studies in various areas of art, design, and technical education oriented towards facilitating creativity.

\section{Function-based Modelling and the HyperFun Project in Education}

In the Function Representation (FRep) (Pasko et al., 1995), a 3D object is represented by a continuous function of point coordinates through the inequality $F(x, y, z) \geq 0$. Time-varying and other multidimensional objects can be defined by a similar inequality. In an FRep modelling system, an object is represented by a constructive tree data structure (Figure 1 left) reflecting the logic of the object construction, where leaf nodes represent primitives (building blocks) and internal nodes represent operations. The function $F$ is evaluated at a given point by an FRep tree postorder traversal procedure. The noticeable advantages of this representation are its procedural nature and extensibility, or the ability to introduce a new primitive or operation via a small analytical expression or a short function evaluation procedure. The research results on various FRep primitives and operations are reported at the FRep Web page (Shape Modelling and Computer Graphics with Real Functions www.hyperfun.org/F-rep.html). Later (Pasko et al., 2001), a more general constructive hypervolume model was introduced, which supports modelling heterogeneous volumetric objects as point sets with attributes, where an attribute is a mathematical model of an object property of an arbitrary nature such as material, photometric, physical, and others. 
There are several projects adapting the FRep modelling paradigm: the general FRep modelling system HyperFun (HyperFun Project, 2012), the skeleton-based implicit surface modelling system BlobTree (Wyvill, Galin, and Guy, 1999), and the function-based extension of VRML and X3D formats called FVRML/FX3D (Liu \& Sourin, 2006). In this paper, we present a number of educational case studies based on various FRep software tools including HyperFun. The members of the HyperFun team, a freely associated group of researchers and students from different countries, have contributed to the case studies described in this paper.

HyperFun (Adzhiev et al., 1999; HyperFun Project, 2012) is a programming language supporting all notions of FRep modelling. This language was designed to be a minimalist one in order to allow non-specialist users to create models of complex geometric shapes. A model in HyperFun (see Figure 2) can be constructed using traditional imperative programming statements such as assignment, iteration, and condition. The functional expressions are presented using arithmetic and relational operators, standard mathematical functions as well as special built-in operators, which support fundamental set-theoretic operations (union, intersection, subtraction), and special FRep library functions.
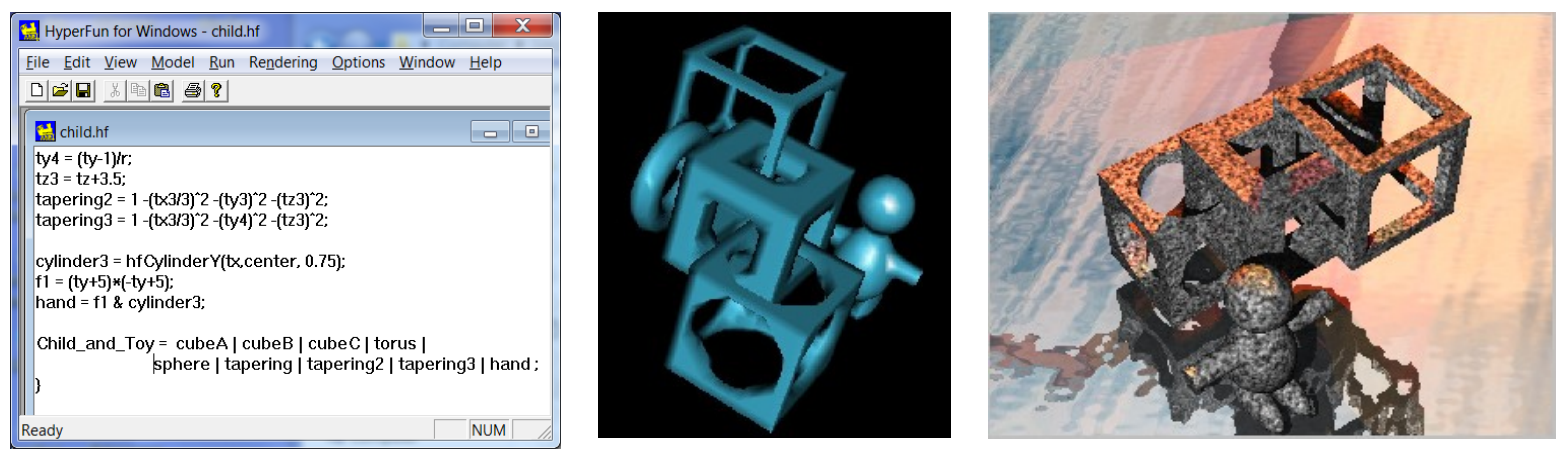

Figure 2: Example of a HyperFun model fragment (left) and the images of the polygonized (middle) and ray-traced (right) model.

The FRep library contains the most common primitives and transformations and is easily extensible. There are functions implementing traditional geometric modelling primitives (block, sphere, cylinder, cone, torus) and special implicit surface primitives (blobby objects, soft objects, metaballs). More advanced primitives include convolution objects with various skeletons, pseudorandom solid noise primitives, and objects defined by parametric splines (cubic and Bézier). The typical transformations such as rotation, scaling, translation, twisting, stretching, tapering, blending are supported as well as more advanced operations such as metamorphosis or non-linear deformations driven by control points. Details of the HyperFun language and associated freely available software tools can be found at the HyperFun Project Web site (HyperFun Project, 2012) and in the corresponding publications (Adzhiev et al., 1999; Cartwright, Adzhiev, Pasko, Goto, \& Kunii, 2005; Pasko \& Adzhiev, 2009).

FRep modelling, HyperFun language, and supporting software tools have been taught at several levels of education starting from elementary schools to the postgraduate university level. The primary target was mathematical education at schools and universities combined with practical experience in modelling, computer graphics, animation, and visualization. More than a thousand school and university students in Japan, Russia, United Kingdom, Norway, France, Slovakia, Austria, Sweden, Albania, and United Arab Emirates studied FRep modelling using HyperFun language in the following courses and exercises: computer graphics, shape modelling, visualization, computer animation, and compiler design. Examples of shape models created by students of different ages and qualifications can be found in the Gallery at the HyperFun Web site (HyperFun Project, 2012). 
Teaching FRep and HyperFun in computing classes can go through several stages. As HyperFun is quite a simple language it can be used for teaching the basics of computer programming. At the low level, all the mathematical and geometric modelling concepts can be hidden from students. At a higher level, the underlying mathematical and geometric concepts can be revealed in the courses of linear algebra and analytical geometry, computer graphics, solid modeling, and other specialist disciplines. Finally, at the highest level, the HyperFun language can be used for modelling shapes with complex mathematical and procedural definitions by graduate and postgraduate students in different research areas. Another aspect of using FRep and HyperFun concepts and software is stimulating imagination and facilitating creativity in educating non-computer specialist users such as artists, designers, and students in natural sciences. The case studies presented in this paper are intended to illustrate this branch of our educational practice.

\section{Case Studies in Art, Design, and Technical Education}

\section{Augmented Sculpture Project}

The Augmented Sculpture project (Adzhiev, Comninos, \& Pasko, 2003) illustrates a more complex and creative multilevel activity within our framework. In this project, we introduced and explored an original approach to computer-based sculpting that can be of interest not only for CG professionals but for art students and artists. The purpose was to develop a specific interactive environment with embedded computer-based means of sculptural representation to produce artifacts with a new aesthetics. Viewers experiencing these shapes within a virtual space can also benefit from this technology. One can start from an existing physical sculpture, then create its computer model and manipulate this model to generate new shapes that can eventually be manufactured to produce a new physical sculpture. We call this approach "augmented sculpting" as it extends the existence of physical artifacts to a virtual world and then closes the loop by bringing new computer models into physical existence.

The photographs of the sculptures by Russian artist Igor Seleznev were made available to computer-graphics students of the National Research Nuclear University "MEPhI" via the Web. To create initially sketchy computer models of the sculptures, the students were encouraged to write collaboratively and share the HyperFun code. They then formed groups of two or three to combine their efforts in developing more accurate models. The copies of the physical sculptures were made available to the students at this stage, and the artist himself took part in assessing and discussing the intermediate results with the students. Finally, the students constructed geometric models of the sculptures in the form of programs in HyperFun language using the HyperFun for Windows toolkit, and then generated the final ray-traced renderings using the HyperFun for POVRay toolkit. Figure 3 (top left) shows just one sculpture "Gymnast", and Figure 3 (top right) shows the ray-traced image of its computer model. Three frames from the animation sequence (implemented by another student who got access to the code of the "Gymnast" model in HyperFun) that brought the "Gymnast" to life are shown in Figure 3 (middle). Note that the sculptures have quite complex shapes with subtle non-regular features, and students could see benefits from using such advanced primitives as convolution surfaces.

Other very interesting sculpting artefacts can be created using a metamorphosis operation. Traditionally, metamorphosis is a complex task that requires the animator to establish a set of correspondence between the initial and final key shapes. In the FRep framework, metamorphosis is performed quite simply by a non-specialist user and can generate intermediate shapes by interpolating between more than two key ones. In the Augmented Sculpture project, students of the Hosei University in Tokyo had received from MEPhI students the Hyperfun code for metamorphosis between three sculpture models ("Gymnast" and two others - "Naked" and "Walking An- 
drogynous") that made it possible to generate a shape that was actually a weighted sum of all those sculptures. The Japanese students then produced the physical incarnations of that shape using rapid prototyping (RP) machinery and the process called '3D printing'. Figure 3 (bottom left) shows three original sculpture models, fabricated using a SLA3500 RP machine. The result of the metamorphosis is shown in Figure 3 (bottom right) fabricated using Laminated Object Manufacturing LOM (KIRA Solid Center).
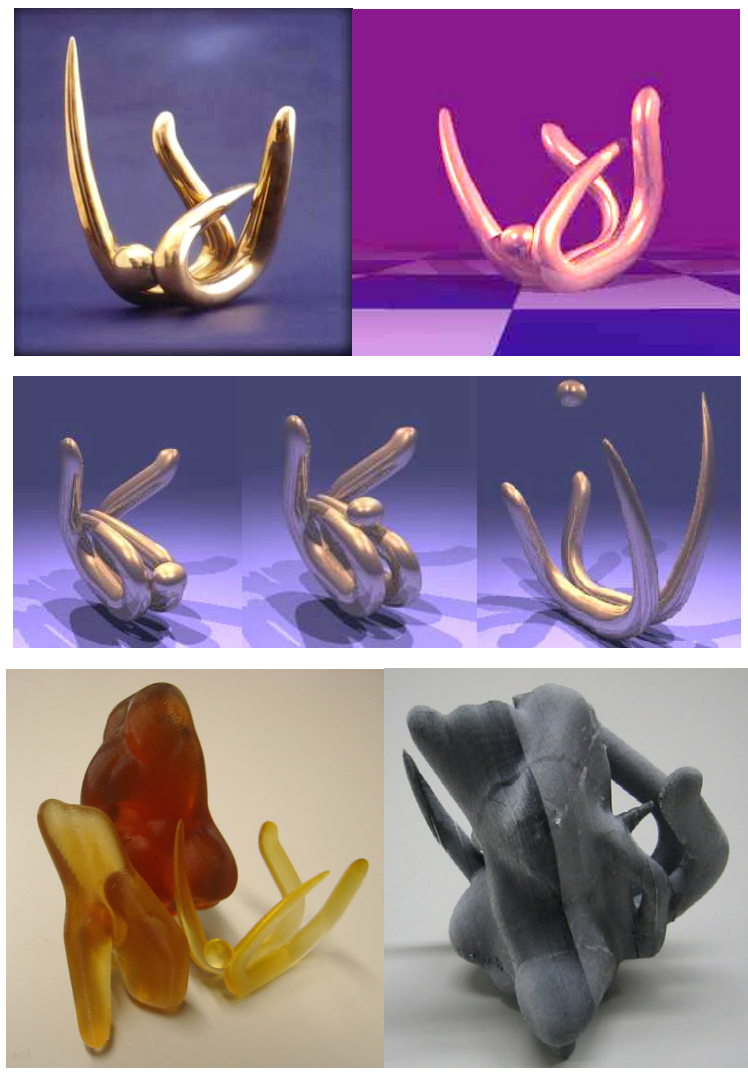

Figure 3: Augmented Sculpture Project:

(top left) real sculpture; (top right) its HyperFun model;

(middle row) frames of the gymnast animation;

(bottom left) models of three sculptures produced by a RP machine;

(bottom right) RP model of the "triangle metamorphosis".

The next stage of the project was concerned with using an experimental interactive modelling system allowing the artist to interactively navigate through a so-called 'FRep Sculpture Garden', which is a time-dependent scene composed of multiple objects. So, the artist experiences an immersion in a virtual space where he or she can generate new shapes using metamorphosis between the sculptures. Editing the shapes on the fly by adding or removing material is also possible. This is a base for an interactive art installation in which physical and virtual artifacts are combined and overlaid (Adzhiev et al., 2003). We believe that projects like Augmented Sculpture are a way of allowing professionals, artists, and students to mix and work together, thus encouraging them to exchange ideas and skills. A more detailed description of the project can be found in Adzhiev et al. (2003). 


\section{"Lifetime" Animation}

The National Centre for Computer Animation (NCCA) at the Bournemouth University is a leading educational and research school that provides courses at undergraduate and graduate level in computer animation, visual effects, and games (Comninos, McLoughlin, \& Anderson, 2010). Its graduates can be found working at major studios worldwide and some Alumni have been awarded Oscars and BAFTAs. On successful completion of "the major project" in the final year, NCCA's undergraduate students have to produce a body of work (e.g., a computer animation artefact) of professional quality mainly using industrial tools, in particular Maya, which is a major mainstream 3D animation software system. It provides a comprehensive feature set that includes tools for animation, modelling, simulation, rendering, and match moving. Those tools are mainly surface-based and do not provide FRep support, so our group has developed an FRep plug-in to Maya thus extending its functionality. Here we present a final year project by a student Paul Novorol who created an animation called "Lifetime" (Novorol, 2011) using this novel tool.

The main idea for the short animation was to create a human life cycle using animated liquid wax based on a lava lamp. The FRep tool allowed the author to handle a smooth flowing anthropomorphic object whose topology updated and changed frame by frame making it possible to form, blend and metamorph into many different organic and complex shapes within the same sequence. This animation could not be implemented using conventional tools, so the student who intended to work in industry as an artist had to learn some novel modelling concepts and master a research software tool. Figure 4 shows four frames from the animation.
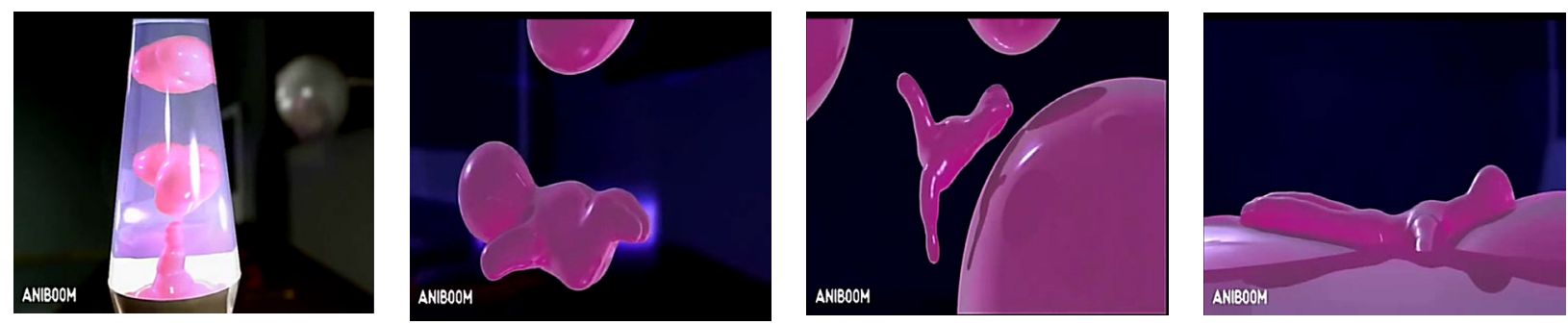

Figure 4: Frames of the "Lifetime" animation.

\section{Kinect FRep Modeller}

The Kinect FRep Modeller (Glynos, Miltiadou, van Mourik, \& Thattarakkal Poothakandy, 2012) was a group project by master's students at the National Centre for Computer Animation. The goal of the project was software development to allow the users to manipulate objects and to perform constructive modelling with their hands rather than using a mouse and a keyboard. Figure 5 illustrates the main concepts of the project. The core of the project technology is built around the Kinect sensor device (Glynos et al., 2012) providing the depth data together with the video signal. This allows for tracking body motion including hand gestures (Figure 5a). In the project, the hand detection is implemented through contours (Figure 5b). The detected hands are represented on the screen for feedback by the blue and red cursors (Figure 5c). A basic hand grab gesture has been implemented to perform such actions as selecting a primitive or operation from the menus, rotating the camera and moving the objects in the scene. A FRep library supporting the constructive modelling (Figure 5d) has been implemented and includes basic geometric primitives and set operations. The current object is rendered using its surface polygonization. Several test models have been produced and the modelling process documented in a video (Glynos et al., 2012). The main intent of this technical project is to provide means for users to express their creativity by using their hand gestures to construct 3D shapes in a game-like environment. 


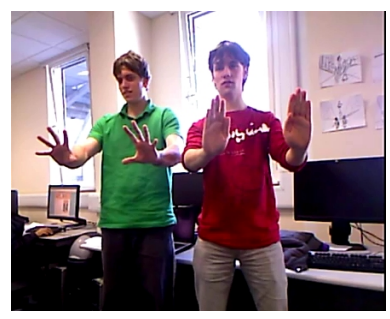

a

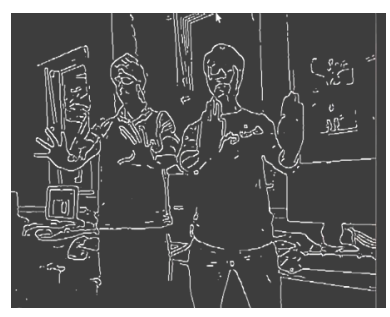

b

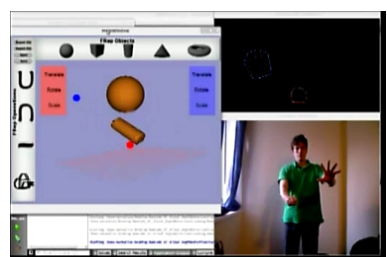

c

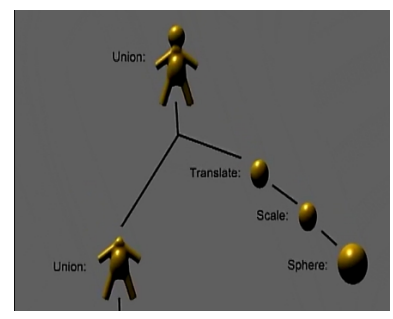

$\mathrm{d}$

Figure 5: Kinect FRep Modeller concepts: a) user control with hand gestures; b) hands detection using a Kinect camera; c) a cursor attached to either hand; d) constructive modelling using the menus of primitives and operations.

\section{Jewelry Design and Fabrication}

In the design school Breivang in Tromsø, northern Norway, students learned how to take their designs from a paper sketch, to a 3D digital model, and then to fabricate a customised piece of silver jewelry (Figure 6). The students were taken through the entire process, starting with the design concept on paper. After a few sessions in 3D software and HyperFun in particular, they transferred their designs from paper to digital 3D models. A wax cast was made for each student's 3D model using a Roland Modela MDX-20 desktop milling machine. Next the students used the special Art Clay material consisting of silver particles and organic binder to fill their casts, which were then dried, fired on a portable kiln, further polished and stylized by the students using traditional jeweller's tools. More details on the technological process can be found at Vilbrandt, Vilbrandt, Pasko, Stamm, and Pasko (2011). Each of the students who participated in the class ended up with a digital model and a real piece of silver jewellery from their original paper design.

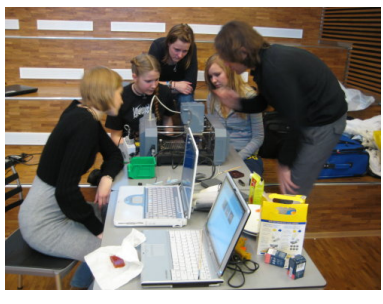

a

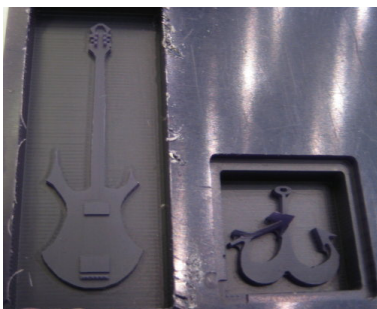

$\mathrm{b}$

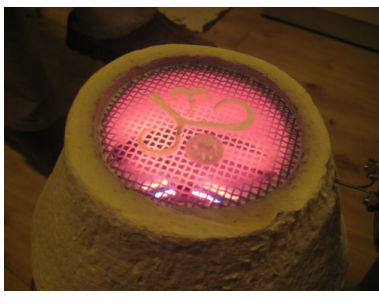

$\mathrm{c}$

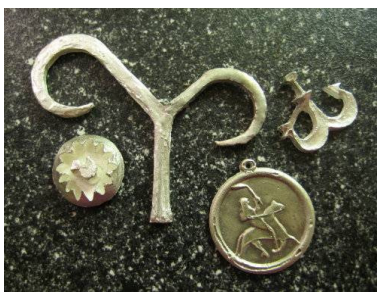

d

Figure 6: The process of silver jewelry fabrication:

a) wax cast producing with Modela milling machine; b) samples of wax casts; c) silver clay objects fired on a portable kiln; d) final silver artefacts.

\section{SHIVA Metamorphosis Exercise}

The EU sponsored SHIVA Project (Sculpture for Health-care: Interaction and Virtual Art in 3D) brings together several computer science, educational, and medical partners from the UK and France. The main idea behind SHIVA is to give people with disabilities an opportunity to do something in the area of 3D modelling as a way of enhancing their creativity and expressing themselves. The project team develops computer-aided exercises to support a range of different patient activities.

One of the developed exercises allows for children with disabilities to perform a metamorphosis transformation between two arbitrarily selected 3D shapes (Figure 7) (Mcloughlin et al., 2012). 
The main design issue for this exercise was the simplicity and adaptivity of the graphical user interface, which is aimed to support the students with very limited motor skills including those operating with a single switch (large push button, lever switch, eye-blink trigger). Typical computer-aided activities used by disabled children include typing text and performing other simple tasks on the 2D surface of the computer screen. Providing them with the ability to operate with $3 \mathrm{D}$ shapes is thought to stimulate their imagination and to bring satisfaction from using the computer. There are several other exercises under development such as axis-aligned and free-form constructive modeling. The project aims to be completed with an exhibition, which will display the sculptures of the disabled students. This will be made possible through the use of desktop 3D printers.

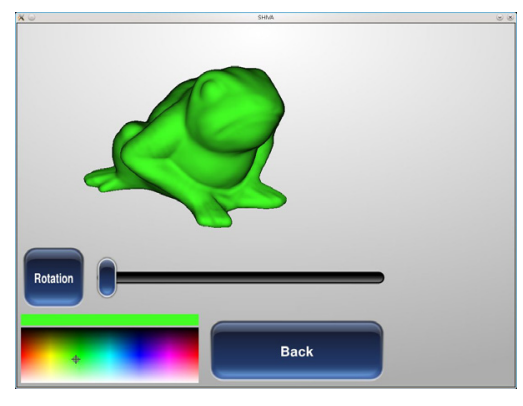

a

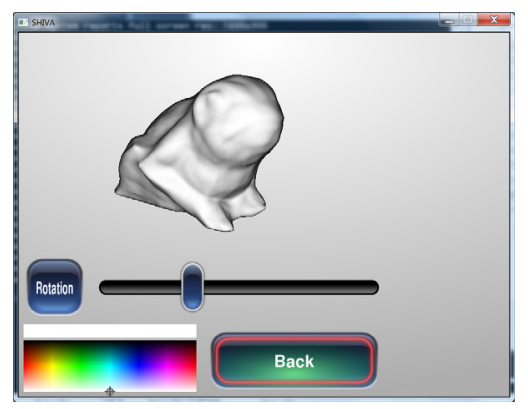

b

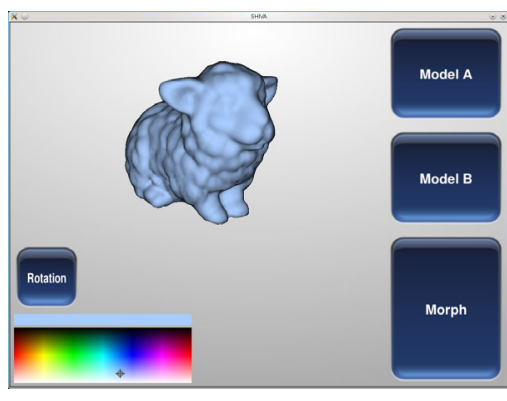

Figure 7: Screeenshots of the 3D metamorphosis exercise of the SHIVA project: gallery shapes ( $a$ and $c$ ) and an intermediate shape (b).

\section{Scientific Visualization in Physics Education}

Nowadays it is especially important for students in physics to have skills in computer modelling physical objects and processes that can be very helpful in their research work. As scientific visualization tools are widely used for analysis of computer modelling results, students in physics are often taught to visualize results of computer modelling as well. We describe here the experience of using HyperFun as a scientific visualization tool for analysis of various physical objects and processes that are the results of computer modelling work done by physics departments at the National Research Nuclear University "MEPhI" (Moscow, Russia). Most frequently, the studied results of computer modelling are physical scalar fields. Students are taught to consider different static and dynamic spatial images of computer modeled scalar fields in the process of their analysis by means of scientific visualization.

The studied scalar fields were given as functions of several variables defined on domains represented as geometric objects that also could be defined by functions of several variables. A functional description of a scalar field and its domain was obtained as the result of computer modelling within students' research work. Such description of the studied physical object was presented in a file in the form of numerical data that should be analyzed. The example of scalar field visualization is shown in Figure 8. To obtain these results several additional file reading based primitives and attribute functions were added to the HyperFun FRep library and used with the resulting HyperFun model. The visualization presented in Figure 8 was made through the Visualization Toolkit (VTK) based interface for HyperFun. These graphical representations of spatial scenes corresponding to the second type superconductor computer modeled with Ginzburg-Landau equations help students to form statements about the physical variable under study, namely the scalar order-parameter field distribution in the selected space area.

One more example is "The Principles of Scientific Visualization" course, which is taught to graduate students in physics at the National Research Nuclear University "MEPhI". This course 
was developed by the "Scientific Visualization" laboratory with the close support of physics departments and the National Centre for Computer Animation, Bournemouth University, UK. The purpose of this course is studying the theoretical principles of scientific visualization and acquisition of practical skills in development of application software in scientific visualization. In general, the course aims to develop the students' spatial creative thinking while solving problems of scientific data analysis. So, this course teaches students to consider and manipulate the corresponding spatial images, make their visual analysis and interpret the analysis results in terms of the application area.

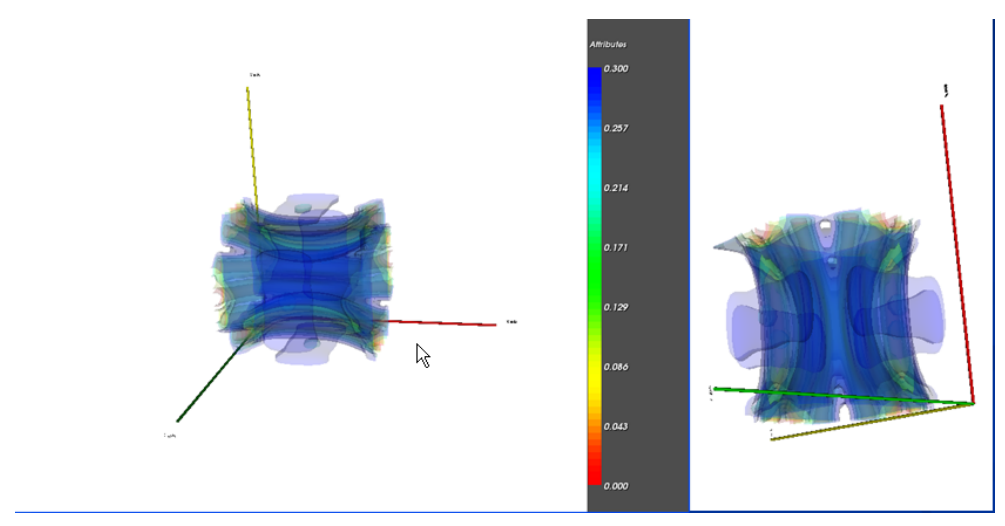

$\mathrm{a}$
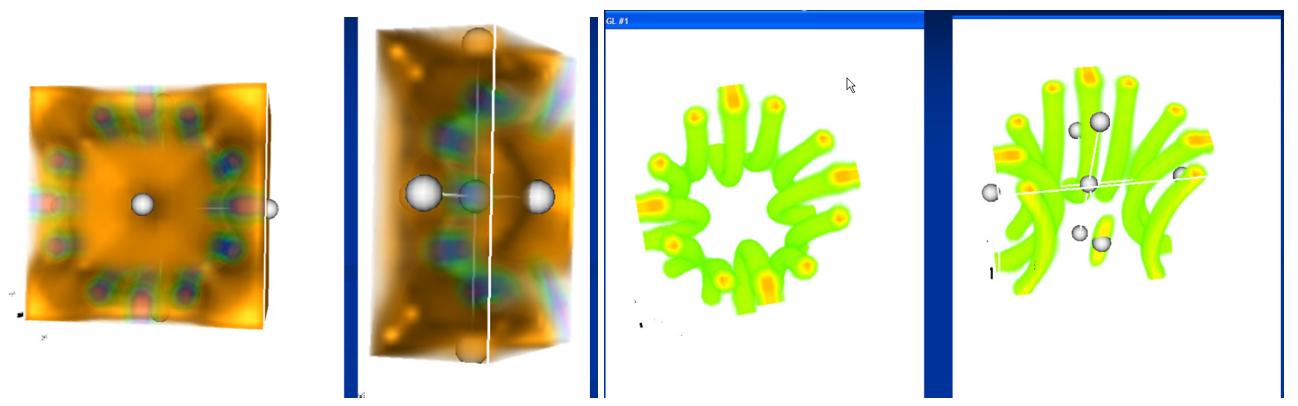

b

c

Figure 8: Scientific visualization example for the order-parameter field for the second type superconductor (Abrikosov vortices). Different approaches to scalar field visualization:

a) isosurfaces; b) volume visualization with the scalar field defined on a rectangular domain;

c) scalar field on a complex domain defined by one of the field's isosurfaces. The field's domain can be interactively cross-sectioned by the user with planes controlled by white handles.

The course includes the following three sections: scientific visualization concepts and capabilities, scientific visualization tools, and scientific visualization applications. The second section includes an introduction to HyperFun as well as other software tools that can be used for solving problems of data analysis by scientific visualization means. Students may write application scientific visualization software on the base of HyperFun in the practical part of the course. To support this, the HyperFun language was extended: the program functions of file opening, closing, reading, writing were added to the HyperFun API. An example of an application software on the base of these functions is given in the second section of the course. It should be mentioned that HyperFun was also used for the course multimedia training materials design that students have an opportunity to use. 


\section{Conclusions}

Constructive function-based geometric modelling can be seen as a highly specialized subject interesting only for professionals. Our experience shows that it can be mastered and appreciated by students at different levels of education as an activity stimulating and supporting their creative thinking.

In the social context of the presented educational technology, we emphasize the active, creative, and collaborative character of the learning process presented in this paper. Indeed, the building of object models starts from rather abstract mathematical expressions, utilizes ready made building blocks in the form of library functions, then goes through interactive modelling in the virtual world, and finally results in the fabrication of physical artefacts. In this process, the learner obtains tangible instances of their creative designs. This can be considered a practical confirmation of the idea of the constructionism theory that learning is most effective when the learner creates a meaningful product actively using their imagination supported by advanced computer technology.

\section{Acknowledgements}

This work was partially sponsored by the EU Interreg IVA project 5-023-FR SHIVA.

\section{References}

Adzhiev, V., Cartwright, R., Fausett, E., Ossipov, A., Pasko, A., \& Savchenko, V. (1999, June). HyperFun project: A framework for collaborative multidimensional FRep modelling, Proceedings of Implicit Surfaces '99, Eurographics/ACM SIGGRAPH Workshop, 59-69.

Adzhiev, V., Comninos, P., \& Pasko, A. (2003, May). Augmented sculpture: Computer ghosts of physical artifacts. Leonardo, 36(3), 211-219. Retrieved from http://www.hyperfun.org/App/ASP/FASP.html

Beynon, M., \& Roe, C. (2004, August). Computer support for constructionism in context. Proceedings of IEEE International Conference on Advanced Learning Technologies 2004, 216 - 220.

Cartwright, R., Adzhiev, V., Pasko, A., Goto, Y., \& Kunii, T. (2005, March). Web-based shape modelling with HyperFun. IEEE Computer Graphics and Applications, 25(2), 60-69.

Comninos, P., McLoughlin, L., \& Anderson, E. F. (2010). Educating technophile artists and artophile technologists: A successful experiment in higher education. Computers \& Graphics, 34(6), 780-790.

Glynos, C., Miltiadou, M., van Mourik, T., \& Thattarakkal Poothakandy, A. (2012). Kinect Frep Modeller, MSc Group Project, National Centre for Computer Animation, Bournemouth University, UK. Available at http://www.youtube.com/watch?v=XVvrsXKxmVE

HyperFun Project. (2012). Language and Software Tools for F-rep Geometric Modelling. www.hyperfun.org

The Kinect patent - Method and system for object reconstruction, (2006). Retrieved from http://patentscope.wipo.int/search/en/WO2007043036

Liu, Q., \& Sourin, A. (2006). Function-defined shape metamorphoses in visual cyberworlds, The Visual Computer, 22(12), 977-990.

Mcloughlin, L., Sanchez, M., Fryazinov, O., Vilbrandt, T., Pasko, A., Adzhiev, V., \& Comninos, P. (2012). Sculpture for Health Care: Interaction and Virtual Art in 3D, SHIVA Project 3rd Progress Report, Bournemouth University, February 2012. www.shiva-project.eu

nOmArch, (2009). Escher in Lego. Retrieved from http://www.fanedit.org/forums/showthread.php? 1896-LE-G-O-real-toys-for-re\%3Eal-men!\&highlight=Lego

Novorol P. (2011). Lifetime, Major project. National Centre for Computer Animation, Bournemouth University, UK. Available at http://www.youtube.com/watch?v=OSYobb76-Sc 
Papert, S., \& Harel, I. (1991). Situating constructionism. In S. Papert \& I. Harel, Constructionism: Research Reports and Essays (pp. 1-11). Ablex Publishing.

Pasko, A., \& Adzhiev, V. (2009). Constructive function-based modelling in multilevel education, Communications of the ACM, 52(9), 118-122.

Pasko, A., Adzhiev, V., Sourin, A., \& Savchenko, V. (1995). Function representation in geometric modelling: Concepts, implementation and applications. The Visual Computer, 11(8), 429-446.

Pasko, A., Adzhiev, V., Schmitt, B., \& Schlick, C. (2001). Constructive hypervolume modelling. Graphical Models, 63(6), 413-442.

Vilbrandt, T., Vilbrandt, C., Pasko, G., Stamm, C., \& Pasko, A. (2011). Digitally interpreting traditional folk crafts. IEEE Computer Graphics and Applications, 31(4), 12-18.

Wyvill, B., Galin, E., \& Guy, A. (1999). Extending the CSG Tree. Warping, blending and Boolean operations in an implicit surface modelling system, Computer Graphics Forum, 18(2), 149-158.

\section{Biographies}

Alexander Pasko is a professor at The National Centre for Computer

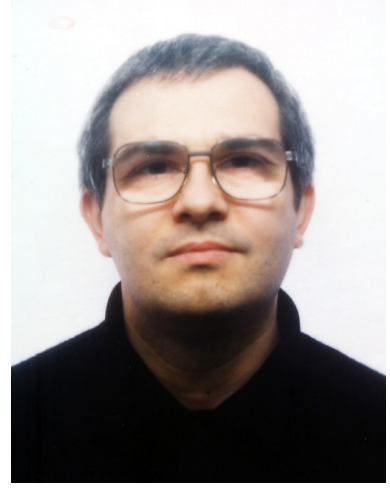
Animation, Bournemouth University, UK. He received his $\mathrm{PhD}$ from Moscow Engineering Physics Institute (MEPI) in Russia in 1988. His main research interest is development of a high-level universal model for spatial objects and phenomena with their internal properties. To support the mathematical concepts of this model, Alexander and his colleagues introduced and develop the special-purpose modeling language called HyperFun (from Hyper-dimensional Functions), which has extensive applications in education, computer animation, biology, digital fabrication, and other areas. Alexander has published more than 120 papers in academic journals and conferences.

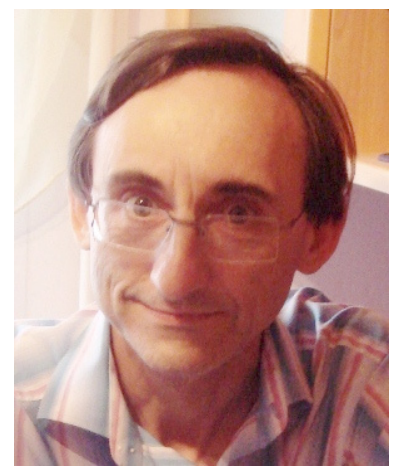

Dr Valery Adzhiev is a senior research lecturer at The National Centre for Computer Animation, Bournemouth University, UK. He received his $\mathrm{PhD}$ in Computer Science from Moscow Engineering Physics Institute (MEPhI) in Russia in 1992. The main focus of his research is on Functionally-based Geometric Modeling and its applications in Computer Animation, Computer Art and 3D Fabrication. He also specializes in programming languages and compilers, especially for geometric modeling and animation applications. He is a designer of HyperFun modeling language. Valery has published more than 70 refereed papers in academic journals, conference proceedings and books. 


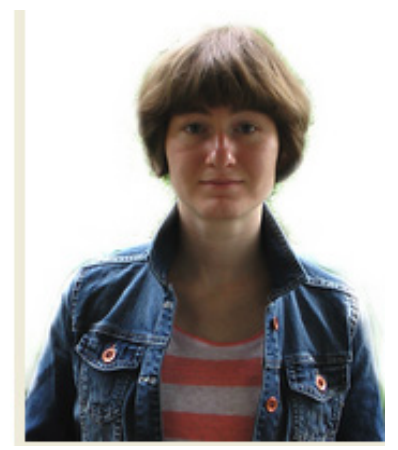

Evgeniya Malikova is currently a $\mathrm{PhD}$ student (National Research Nuclear University "MEPhi", Moscow, Russia). She received her Master Degree in Computer Science in 2009 with the thesis 'Development of multidimensional geometry and visualization tools in Autodesk $3 \mathrm{ds}$ Max'. Her area of experience is multidimensional geometric modelling and rendering using real-valued functions.

Evgenia is also an editorial assistant of the Scientific Visualization Journal and the Computer Graphics \& Geometry Journal ( http://svjournal.com, http://cgg-journal.com), a senior researcher in the National Technical Physics and Automation Research Institute (VNIITFA), Russia, and a software developer and consultant in Uformia AS, Norway.

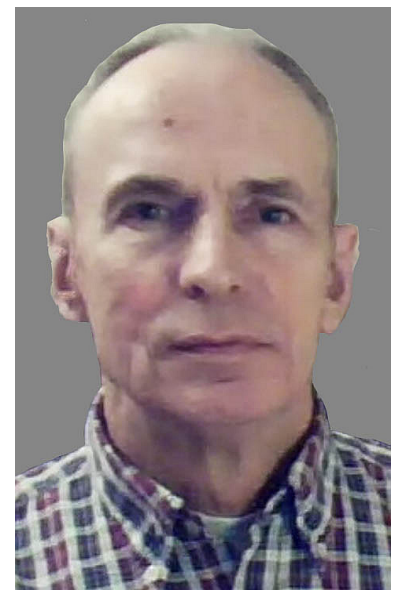

Victor Pilyugin is a professor of the System Analysis department at the National Research Nuclear University "MEPhI" and a head of the University Scientific Visualization Laboratory, Russia. He received his $\mathrm{PhD}$ from Moscow Engineering Physics Institute in Russia in 1975. He is the Scientific Secretary of the "Scientific Visualization" Journal Editorial Board. His research interests include scientific visualization and visual analytics, spatial images mentality by means of computer, geometric modeling and applications. Members of his Scientific Visualization Laboratory developed a number of various application programs, which are widely used in research and education at the National Research Nuclear University "MEPhI" and other organizations. 